\title{
Fuzzy Logic based Approach for VoIP Quality Maintaining
}

\author{
Mohamed E. A. Ebrahim, Hesham A. Hefny \\ Computer and Information Science Department \\ Institute of Statistical Studies and Research (ISSR) \\ Cairo University \\ Giza, Egypt
}

\begin{abstract}
Voice communication is an emerging technology and has great importance in our routine life. Perceptual, Voice over Internet Protocol quality is an important issue for VoIP Apps services because VoIP Apps require real-time support. Many network factors (packet loss, packet delay, and jitter) affect to VoIP quality, to achieve this objective we used an approach based on Fuzzy Logic. We configure Resource Reservation Protocol application to control Token Bucket Algorithm and the simulation experiments are carried out with Opnet. In addition, compare Token Bucket with and without Quality of Service for measure network factors. In this paper, building Fuzzy Token Bucket System consists of three variables (Bandwidth Rate, Buffer Size, and New Token) in order to improve Token Bucket Shaper output variable (New Token) by Fuzzy Stability model for Voice over IP quality maintaining.
\end{abstract}

Keywords-Voice over Internet Protocol (VoIP); Fuzzy model System (FMS); Fuzzy Token Bucket Algorithm (FTBA); Resource Reservation Protocol (RSVP); Quality of Service (QoS)

\section{INTRODUCTION}

VoIP (pronounced as voyp), also known as IP Telephony, is the real-time transmission of voice signals using the Internet Protocol (IP) over the public Internet or a private data network. In simpler terms, VoIP converts the voice signal from your telephone into a digital signal that travels over the Internet as in [1]. (RSVP) Resource reservation is a network protocol that enables IP-based applications to obtain particular (QoS) for data flows. It should be considered as a protocol that delivers QoS requests to the nodes along the data flow path by maintaining appropriate states in these nodes to provide the requested service as in [2], [15]; therefore, VoIP uses a combination of Real-Time Transport Protocol and User Datagram Protocol over IP. UDP, an unreliable service provides no guarantees for delivery and no protection from duplication using IP to transport messages between endpoints in an Internet. RTP, used in conjunction with UDP, provides end-to-end (ETE) network transport functions for applications transmitting real-time data, such as video or voice Apps over network services as in [3], [17]. (RSVP) Resource reservation uses Token Bucket algorithm to maintain QoS attribute. In this paper, we simulate Token Bucket with QoS attribute and compare results by Token Bucket without QoS attribute then use Fuzzy Token Bucket to enhance this scenario. We use Fuzzy logic to get the way to solve the problems facing VoIP to this day and focused on one these problems Token Bucket in order to improve them. The authors used different ways to improve VoIP, we used RSVP protocol for Token Bucket with applying on Matlab for Fuzzy Logic by using Opnet program results for realtime, and this is what distinguishes our paper from the rest of the articles.

This paper is organized as follows: Section 2 provides an overview of related work. Section 3 shows VoIP Based Token Bucket Rate Section 4 presents fuzzy logic based Token Bucket models. Section 5 presents the proposed models to Comparison of results with and without FL. Section 6 describes the simulation environment. Section 7 discusses the derived results. Section 8 presents the conclusion and the future work.

\section{RELATED WORK}

Many types of research had been done for overcoming challenges VoIP QoS, in order to improve performance QoS. They had used different simulators to achieve their goal. In [4], the authors adapted VoIP schemes based on Adaptive multi-rate codec mode to match voice quality to available network bandwidth, the authors focus on using fuzzy logic with the Adaptive multi-rate codec to enhance Priority QoS.

Fuzzy token bucket scheme is compared with token bucket scheme based on two parameters: Average Delay and Throughput was presented in [5], for high-speed ATM networks.

The proposed to carry voice calls over IP networks can generate network congestion due to the weak supervision of the traffic-incoming packet, queuing and scheduling. The authors of [6] presented an approach for using the fuzzy inference system to classify the queuing incoming packet (voice, video, and text); that can reduce recursive loop and starvation.

The authors of [7] used a real-time fuzzy algorithm to estimate the strength of the line echo component of the voice quality in VoIP networks with using Fuzzy Logic to maintain a high level of MOS value in cases of network congestion. 
The authors of [8] used four types of different mechanisms, Jump Window, Exponentially Weighted Moving Average, Leaky Bucket and modified Fuzzy Leaky Bucket techniques to be identified, analyzed and simulated by the traffic parameters for peak rate, mean rate and the burst time, which characterize the source behavior.

In [17], improve the quality of signal transmission in video surveillance system based on IP network, the authors focus on one parameter bandwidth only to reduce the time delay of the data packets.

The proposed to introduce a new class of forwarding error correction (FEC) codes for VoIP communications which support different recovery delay depending on the channel conditions. The authors of [18] presented to Experiments over real-world packet traces further show performance gains of DD codes in terms of perceptually motivated ITU-T G.107.E-model.

Optimization of VoIP network performance based on voice call routing and network reorganization the authors of [16] used reorganization to meet the requirements of VoIP networks deployment at their base has been proposed and focus on VoIP algorithm.

The authors have made an excellent effort to improve performance VoIP QoS; we participated in the effort to reach a new research point by using fuzzy logic based on different rules for three scenarios to reach the best results not achieved in previous works. This work presents a set of ideas combined as simulate VoIP network with and without QoS based on RSVP Protocol by Opnet and configure RSVP and building fuzzy interface system (FIS) based on three variables by Matlab and compare results as in Section 5.

\section{VOIP BASED TOKEN BUCKET RATE}

Attributes describing Resource Reservation Protocol (RSVP) Parameters set by the application are defined in two objects: The QoS Attribute Configuration object and the Application Attribute Configuration object. To run an RSVP simulation, both objects must be included in the scenario. Bandwidth (bytes/sec) specifies the amount of traffic generated by the application at the IP level (including TCP/UDP and IP headers). This value is set to be the token bucket rate in flow specification of Path and Reservation messages. When the reservation is made using this flow specification, this value is set as the reserved bandwidth for the session according to [9], [15].

Because the controlled load service does not precisely control packet delay, any device implementing the controlled load service should not penalize bursts of packets from an application. It should be possible to buffer bursty data. The amount of data that should be buffered can be configured using the Buffer Size (bytes) attribute. This value is used as "bucket size" and is set in Path or Reservation messages for the session. When a reservation is made, the Buffer Size value is the size of the buffer created for data of a particular flow (each queue in the implementation is defined by a bandwidth and buffer size). The amount of data traffic sent over all time periods should not exceed $\mathrm{r} * \mathrm{~T}+\mathrm{b}$ where $\mathrm{r}$, is the token bucket rate which is the value of Bandwidth attribute, b is token bucket size which is the value of Buffer Size, and $\mathrm{T}$ is the measurement interval in seconds as in Opnet modular documentation, 2014 [10], [ 3].

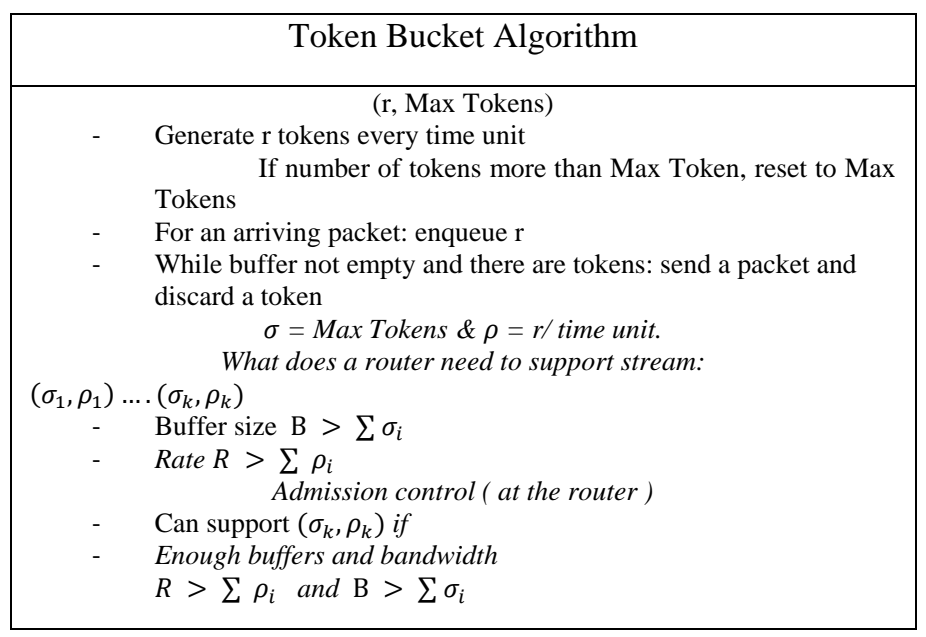

\section{FuZzy Logic BASEd TOKEN BUCKet Models}

The fuzzy logic predictor predicts the token bucket rate required based on the average buffer size rate and available bandwidth rate according to [11]. The fuzzy inputs are the Average Buffer Size Rate 'BufferSize' and Available Bandwidth Rate 'BandwidthRate'. The output is the New Token Bucket Rate denoted by 'NewToken'.

The linguistic values are given below: $\mathrm{VH}\}$

$$
\text { BufferSize }=\{\mathrm{VL}, \mathrm{L}, \mathrm{M}, \mathrm{H}, \mathrm{VH}\}
$$

BandwidthRate $=\{\mathrm{VL}, \mathrm{L}, \mathrm{AL}, \mathrm{BA}, \mathrm{AV}, \mathrm{AA}, \mathrm{BH}, \mathrm{H}$,

NewToken $=\{\mathrm{VL}, \mathrm{L}, \mathrm{BA}, \mathrm{AV}, \mathrm{AA}, \mathrm{H}, \mathrm{VH}\}$

Where, the input BufferSize variable is divided into five fuzzy subsets as shown in Table I.

TABLE I. FIS BUFFERSIZE PARAMETERS

\begin{tabular}{|l|l|}
\hline VL & Very Low \\
\hline L & Low \\
\hline M & Medium \\
\hline H & High \\
\hline VH & Very High \\
\hline
\end{tabular}

These values are normalized in the range of $[0,1]$. We use triangular membership functions, S-shaped and Zshaped functions based on the simplicity of these kinds of functions as Mathworks, 2016 [12] mentioned, is as in (1) and Fig. 1. Triangle-Shaped Membership Function:

$$
f(x ; a, b, c)^{`}=\max \left(\min \left(\frac{x-a}{b-a}, \frac{c-x}{c-b}\right), 0\right)=
$$

Where, the parameters a and c locate the "feet" of the triangle and the parameter b locates the "peak". 


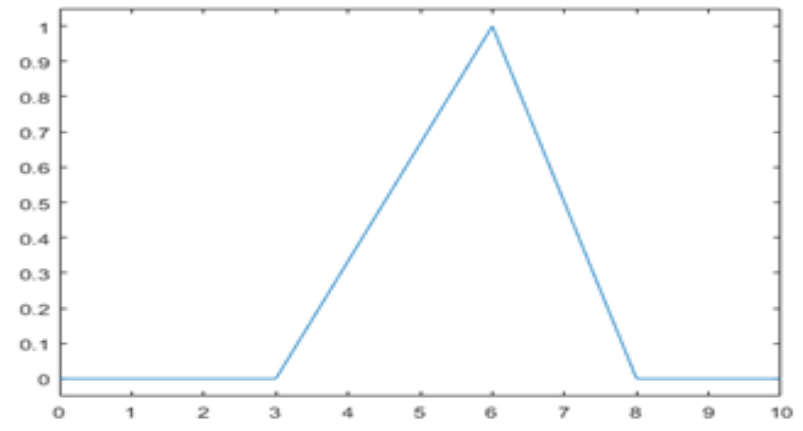

Fig. 1. Triangle trimf, $\mathrm{P}=\left[\begin{array}{lll}3 & 6 & 8\end{array}\right][12]$.

After declaring input and output variables, the membership functions are plotted i.e. the range is defined between 0 and 1 in a normalized form according to [13], [5].

There are five membership functions for FIS BufferSize variable. The value of the variable is increasing from very low to very high. The lower value is $\mathrm{VL}$ and the higher value is $\mathrm{VH}$. Therefore, the name of the variable is given according to the strength of variable. These are shown in Table II as in [8].

TABLE II. MEMBERSHIP FUNCTION PARAMETERS

\begin{tabular}{|c|c|c|}
\hline Name & Type & Parameter \\
\hline VL & zmf & 00.25 \\
\hline L & trimf & 0.00 .250 .5 \\
\hline M & trimf & 0.250 .50 .75 \\
\hline H & trimf & 0.50 .751 \\
\hline VH & smf & 0.751 \\
\hline
\end{tabular}

As shown in Fig. 2, the input variable 'BufferSize' has five membership functions. 'BandwidthRate' was described in Fig. 3, nine membership functions and in Fig. 4, the output variable 'NewToken' has seven membership functions which are of three types: Membership function VL is of $\mathrm{Z}$ - shaped, membership function $\mathrm{VH}$ is of $\mathrm{S}$-shaped and from $\mathrm{L}$ to $\mathrm{H}$ triangular shaped membership function. The input and output variables also have different membership functions. The linguistic variables are those variables whose values are words rather than numbers. Much of fuzzy logic may be viewed as a methodology for computing with words rather than numbers according to [14], [ 8].

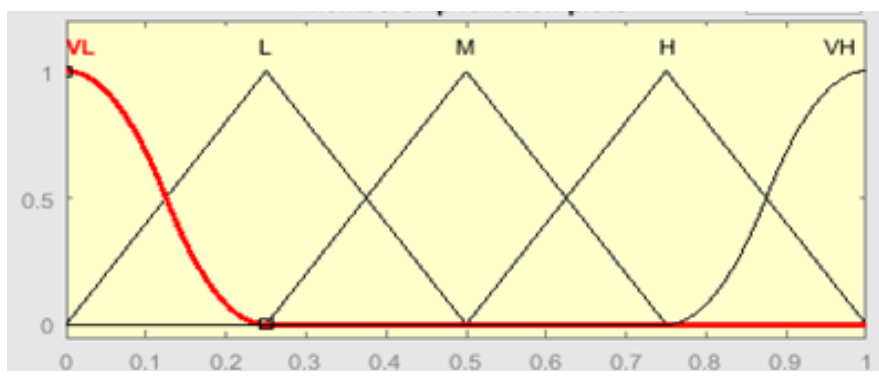

Fig. 2. Membership function for BufferSize.

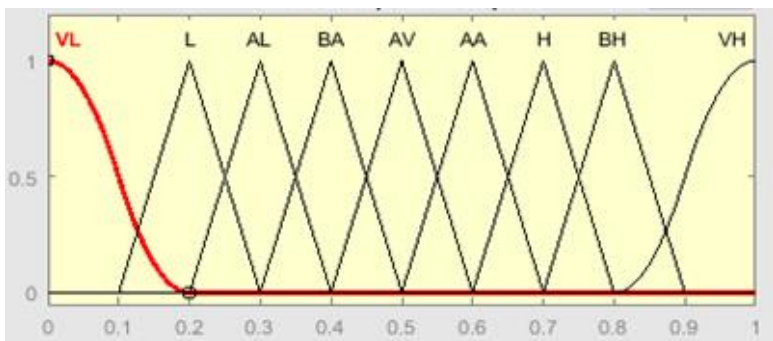

Fig. 3. Membership function for bandwidth rate.

Use Fuzzy sets and fuzzy operators as the subjects and verbs of fuzzy logic to form rule. These "if-then rule" statements are used to formulate the conditional statements that include FL. A single fuzzy if-then rule i.e. assumes the form If (BufferSize is L) and (BandwidthRate is $\mathrm{VL}$ ) then (New Token is $\mathrm{VL}$ ); where $\mathrm{L}$ and $\mathrm{VL}$ are linguistic values defined by fuzzy sets on the ranges (universes of discourse) Low and Very Low respectively. The if-part of the rule "BufferSize is $L$ and BandwidthRate is VL" is called the antecedent or premise, while the then part of the rule "NewToken is VL" is called the consequent or conclusion.

The fuzzy engine has 45 rules that relate the two inputs with the fuzzy output. The constructions of the rules are based on logical reasoning of how the system can track bandwidth usage. It is the normalized form of inputs and output according to [20].

TABLE III. FUZZY CONDITIONAL RULES FOR THE POLICER

ruleBlock->addRule(fl::Rule::parse("if BandwidthRate is $\mathrm{M}$ and BufferSize is VL then NewToken is L",engine));

ruleBlock->addRule(fl::Rule::parse("if BandwidthRate is M and BufferSize is L then NewToken is L", engine));

ruleBlock->addRule(fl::Rule::parse("if BandwidthRate is $\mathrm{H}$ and BufferSize is $\mathrm{L}$ then NewToken is M", engine));

ruleBlock->addRule(fl::Rule::parse("if BandwidthRate is $\mathrm{H}$ and BufferSize is

$\mathrm{H}$ then NewToken is H", engine));

ruleBlock->addRule(fl::Rule::parse("if BandwidthRate is VH and BufferSize is $\mathrm{L}$ then NewToken is $\mathrm{H}^{\prime}$,engine));

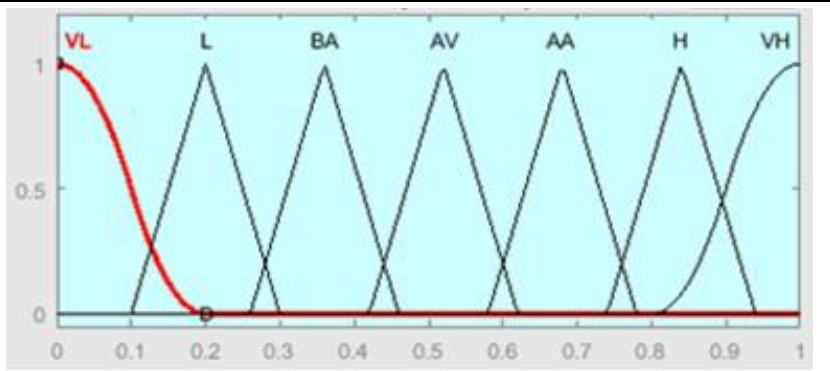

Fig. 4. Membership function for NewToken.

It is observed that the $\mathrm{z}$-axis variable smoothly varies from 0 to 1 , i.e., the new token generation rate is varying in continuous form. In a token bucket with threshold scheme, there was a sudden change in the token generation rate, and this problem is overcome in fuzzy token bucket scheme. Hence this fuzzy token bucket scheme works more efficiently than token bucket scheme. Fuzzy conditional rules for the policer are given in Table III. 


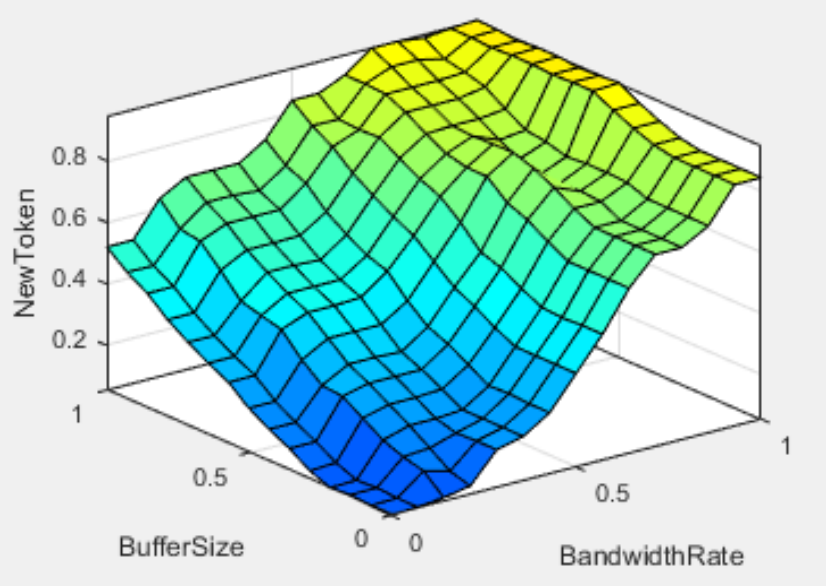

Fig. 5. Surface viewer fuzzy token bucket.

From Fig. 5, the fuzzy token bucket is clear that whenever increase bandwidth rate and buffer size rate the result new token bucket high quality. We conclude from this the strength Fuzzy Token Bucket Shaper depends on bandwidth rate and buffer size rate, and thus lower rate Packet Delay, jitter and highest quality scale Mean Opinion Score (MOS). And here we have succeeded in experience Fuzzy logic to define the criteria on which depend for maintaining quality voice over internet protocol.

\section{COMPARISON OF RESUltS WITH AND WITHOUT FL}

The proposed fuzzy models were tested by OPNET Modeler 14.5 and MATLAB R2016a fuzzy toolbox. Three scenarios were used, first scenario VoIP with QoS, second scenario VoIP without QoS, and third scenario compare the previous result with VoIP based fuzzy logic token bucket. After what we succeeded in identifying the causes of weakness Token Bucket Shaper, we assume Bandwidth Rate and Buffer Size in perfect condition. The proposed explain different factors End-to-End Delay, Delay Variation, Received and sent packet traffic and Mean Opinion Score MOS scale between VoIP with and without Token Bucket and VoIP with fuzzy Token Bucket as in ITU-T Recommendation [19].

In this step, use high Bandwidth Rate and Buffer Size for getting New Token is performed well and work outstanding as shown in Fig. 6.

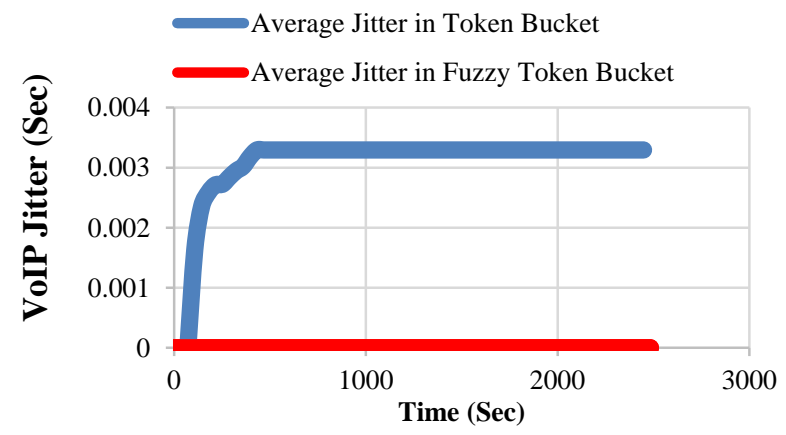

Fig. 6. Comparison of jitter with and without fuzzy logic.
JITTER $=(\mathrm{T} 4-\mathrm{T} 3)-(\mathrm{T} 2-\mathrm{T} 1)$

Fig. 6 compares the Jitter by using a fuzzy token bucket and non-fuzzy token bucket, the time is shown on $\mathrm{x}$-axis and Jitter rate is shown at the $\mathrm{y}$-axis. Blue line depicts Average Jitter in a token bucket and red line depicts the Jitter by the fuzzy token bucket. Therefore, the fuzzy token bucket is performed very well compared with a non-fuzzy token bucket.

Fig. 7 compares the Delay variation by using a fuzzy token bucket and token bucket with and without QoS. The time is shown on the $\mathrm{x}$-axis and Delay variation rate is shown at the y-axis. Blue line depicts Delay variation with a token bucket, brown line depicts Delay variation without QoS, and red line depicts the Delay variation by the fuzzy token bucket. The result is no Delay variation by the fuzzy token bucket.

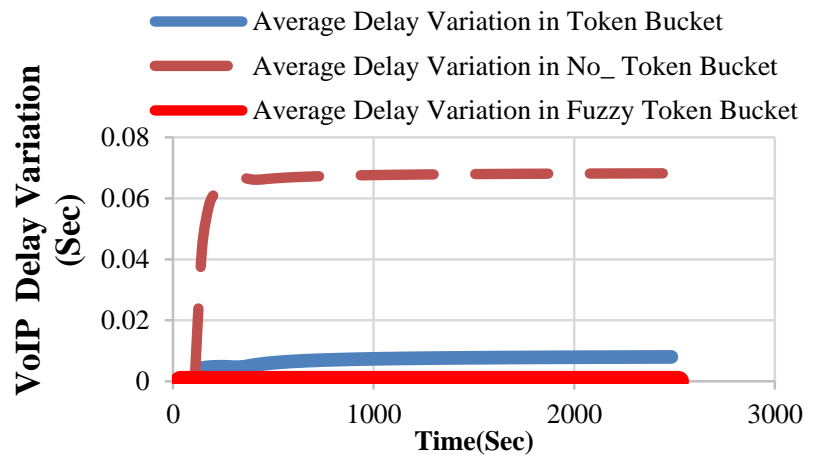

Fig. 7. Comparison of delay variation with and without fuzzy logic.

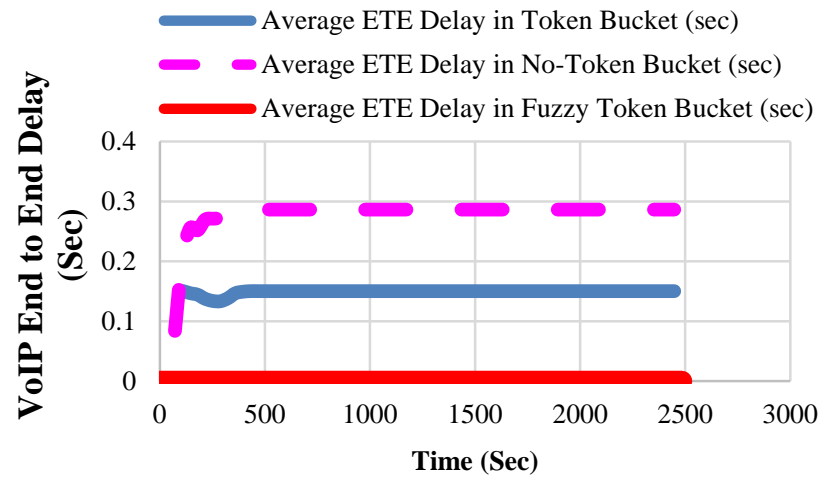

Fig. 8. Comparison of end-to-end (ete) delay with and without fuzzy logic.

\section{DELAY $=($ NETWORK_DELAY + ENCODING_DELAY + DECODING_DELAY + COMPRESSION_DELAY + DECOMPRESSION_DELAY)

Fig. 8 compares the total voice packet delay called End-to-End Delay by using a fuzzy token bucket and token bucket with and without QoS, the time is shown on the $\mathrm{x}$-axis and End-to-End Delay rate is shown at the $\mathrm{y}$ axis. Light Blue line depicts Delay variation with a token bucket, Fuchsia line depicts End-to-End Delay without QoS, and red line depicts the End-to-End Delay by the fuzzy token bucket. The result is no ETE Delay by the fuzzy token bucket. 
Fig. 9 compares the Mean Opinion Score (MOS) by using a fuzzy token bucket and token bucket with and without QoS, the time is shown on the $\mathrm{x}$-axis and MOS scale is shown at the y-axis. Red line depicts MOS by the fuzzy token bucket, Purple line depicts MOS with a token bucket, and Blue line depicts MOS without QoS. The result is high accuracy for VoIP.

Fig. 10 compares the data loss by using a fuzzy token bucket and non-fuzzy token bucket the time is shown on $\mathrm{x}$-axis and data loss rate is shown at the $\mathrm{y}$-axis. Blue line depicts data loss with a token bucket and red line depicts the data loss by the fuzzy token bucket. The result is no Packet Loss by the fuzzy token bucket.

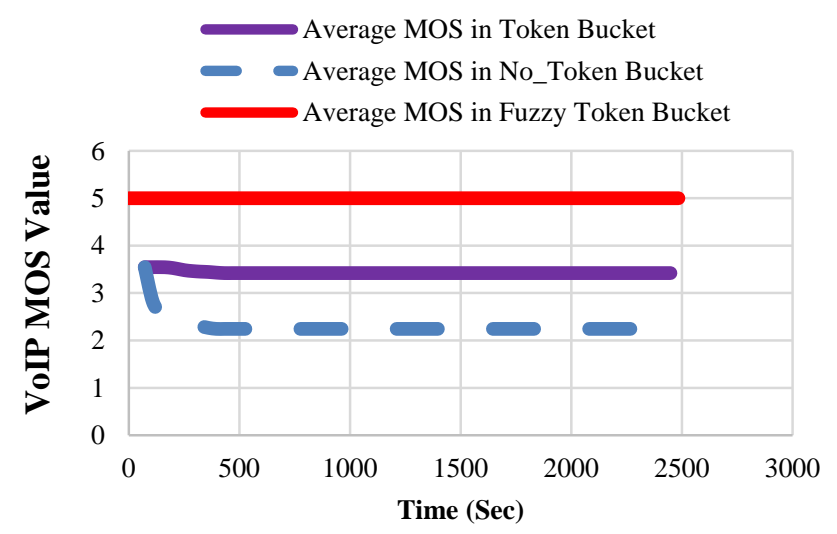

Fig. 9. Comparison of MOS with and without fuzzy logic.

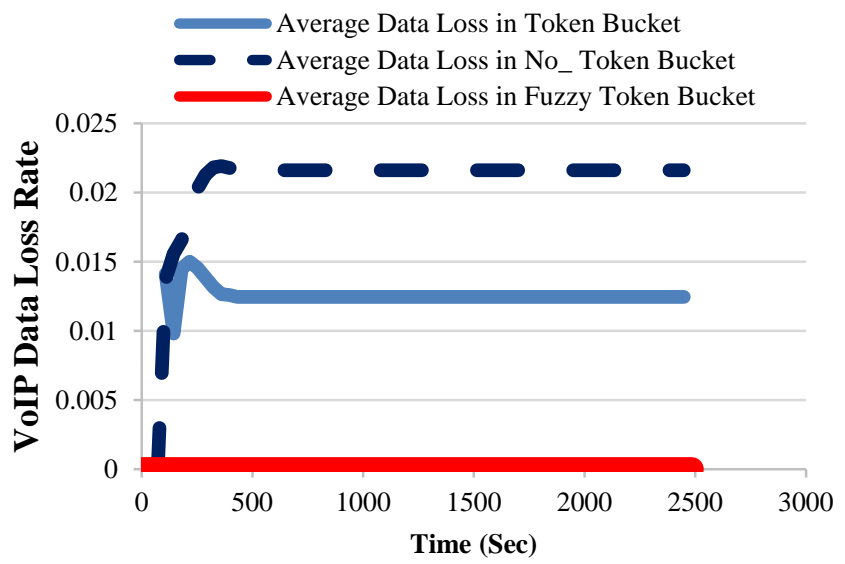

Fig. 10. Comparison of data loss with and without fuzzy logic.

\section{DISCUSSION}

In Fig. 6 comparison between Average Jitter by using a fuzzy token bucket and non-fuzzy token bucket as is evident in the graph. Use fuzzy model has a clear effect on the new token bucket and give rise to zero rates of Jitter. In addition, try another test fuzzy logic token bucket with delay variation parameter in Fig. 7. The result was in favor of fuzzy token bucket after comparison of Delay variation with and without QoS token bucket then add a fuzzy token bucket. Then we repeated another comparison by the end-to-end delay in Fig. 8. The result was clear in graph packet ETE Delay in fuzzy token bucket much better than packet ETE Delay in a token bucket without a fuzzy model. In Fig. 9 we compare the Mean Opinion Score (MOS) by using a fuzzy token bucket and token bucket with and without QoS The result in the graph is high score 5 value in favor of fuzzy token bucket while QoS token bucket is 3.4 value and token bucket without QoS is 2.2 value and also in Fig. 10. We use packet Data loss parameter for comparison data loss in a fuzzy token bucket and non-fuzzy token bucket, the result was no data loss with a fuzzy token bucket, and here it clearly shows that fuzzy model has an effect on token bucket algorithm shaper. We have succeeded in proving Fuzzy logic define the criteria on which depend for maintaining quality voice over internet protocol (VoIP).

\section{CONCLUSIONS AND FUTURE WORK}

This thesis has discussed a proposed approach for improving VoIP quality. The Objective of thesis was developed an approach for VoIP QoS, we used fuzzy logic control system for improve QoS by apply fuzzy token bucket and depend on Fuzzy Stability model (FTBS) to determine the optimal way to get new token bucket do not cause any factors as (jitter - Delay - packet loss) and compare with multi scenarios for parameters Jitter, End to End Delay, Delay Variation, packet traffic and Mean Opinion Score MOS scale between VoIP with and without QoS and VoIP with fuzzy Token Bucket. And was the result of research whenever improving bandwidth rate and buffer size rate the result a new token bucket at the same rate of improvement and vice versa. Therefore, Bandwidth should be sufficient for traffic since insufficient bandwidth may decrease QoS for the flow and buffer size also. Future work will be to analyze the working of VoIP on mobile 5th generation internet. The performance of fuzzy predictor for packet traffic rate in 5th generation mobile networks will also be a future scope of the project.

\section{ACKNOWLEDGMENT}

First and foremost, I would like to express my deepest sense of gratitude to my supervisor Prof. Dr. Hesham Ahmed Hefny who has been supporting my studies using his vast knowledge and skill in many areas, guiding my thesis and constant involvement in guiding me towards my goal, also for a lot of kindness, patience and effectual support. My search could not have been finished reasonably without insightful advice from him.

My thanks and appreciation to all other ISSR professors for many things I learned from them during my study in ISSR.

Finally, I'm deeply grateful to my family, especially my wife, and my sons for their love, support, and patience during writing the thesis.

\section{REFERENCES}

[1] Joe Hallock, "A Brief History of VoIP" Evolution and Trends in Digital Media Technologies-COM538, University of Washington. $26^{\text {th }}$ November 2004.

[2] Nicos A. Antoniou, "Experimental Study of RSVP over ATM" San Diego State University, 2001 
[3] Chintan Vaishnav, "Voice over Internet Protocol (VoIP): The Dynamics of Technology and Regulation" The Massachusetts Institute of Technology, USA, $12^{\text {nd }}$ May 2006.

[4] E. Jammeh, I. Mkwawa, L. Sun, E.Ifeachor, "Type-2 fuzzy logic control of PQoS driven adaptive VoIP scheme" Electronic Letters.46(2), United Kingdom $21^{\text {st }}$ January 2010.

[5] Anurag, A. "Fine Tuning of Fuzzy Token Bucket Scheme for Congestion Control in High-Speed Networks" In: IEEE Second International Conference on Computer Engineering and Applications, 1, pp. 170-174. March 2010.

[6] Suardinata, Bin Abu Bakar, N.Suanmali, N. "comparison process long execution between PQ algorithm and new fuzzy logic algorithm for VoIP" International Journal of Security and Its Application (IJNSA), 3 (1), January 2011.

[7] Oyetade Durojaiye, Elizabeth. N. Onwuka, "Voice Quality Evaluation of a Call Using Fuzzy Logic" International Journal of Network and Communication, 2(2): 7-12, 2012.

[8] Ming-Chang Huang, Seyed Hossein Hosseini, K.Vairavan, and Hui LAN, "Fuzzy Congestion Control and Policing in ATM Networks" International Journal of Engineering (IJE), 3(1), 2012.

[9] Lixia Zhang, Stephen Deering, Deborah Estrin, Scott Shenker, Daniel Zappala, "RSVP: A New Resource Reservation Protocol" IEEE Network Magazine, September 1993.

[10] Opnet modular documentation, 2014.

[11] Demet Dilekci, Conrad Wang, Jiang Feng Xu. "The Analysis and Simulation of VoIP" ENSC 427 Communication Networks, Spring 2013.
[12] http://www.mathworks.com/help/fuzzy/trimf.html

[13] Anurag Aeronl, C. Rama Krishna1, Mohan Lal. "Performance Evaluation of Fine Tuned Fuzzy Token Bucket Scheme for High-Speed Networks" CCSIT, Part2, CCIS 132, PP. 126-136 Springer-Verlag Berlin Heidelberg 2011.

[14] R. Alcal'a, J. Casillas, O. Cord’on, F. Herrera, S. J. I. Zwiry, "Techniques for Learning and Tuning Fuzzy Rule-based system for Linguistic Modeling and their Application" CICYT TIC96-0778 Spain 2010.

[15] Esmat Mirzamany, Aboubaker Lasebae, and Orhan Gemikonakli "Using aggregated RSVP in nested HMIPv6" IEEE Conference Publications, 2012.

[16] Alexander Soloviev, Victor Bondarenko, "Optimization of VoIP network performance based on voice call routing and network reorganization" IEEE, 2017.

[17] Zhou Lin, Chen Yingmei, Li Zhen, He Zhuzhen, "An Improved Video Monitoring System Based on RSVP Protocol" IEEE, 2015.

[18] Ahmed Badr, Ashish Khisti, Wai-tian Tan, Xiaoqing Zhu, John and G. Apostolopoulos, "FEC for VoIP using dual-delay streaming codes" IEEE, 2017.

[19] ITU-T International Telecommunication Union Recommendation: Traffic control and congestion control in B-ISDN Section 7.2.7, defines traffic shaping as a traffic control mechanism, I.371, March 2004.

[20] Hamdy, A.M., Sayedahmed, Hefny, H.A., Fahmy, I.M.A. "Improving Multiple Routing in Mobile Ad Hoc Networks Using Fuzzy Models" Springer International Publishing AG 2018. Conference on Advanced Intelligent Systems and Informatics 2017, Advances in Intelligent Systems and Computing 639. 2017. 